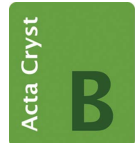

STRUCTURAL SCIENCE CRYSTAL ENGINEERING MATERIALS

ISSN 2052-5206

Received 3 February 2016

Accepted 2 April 2016

Edited by M. Dusek, Academy of Sciences of the Czech Republic, Czech Republic

Keywords: Z' superstructure; hydrogen bonding; superspace; commensurately modulated structure.

CCDC reference: 1472105

B-IncStrDB reference: 11752ErO4ol

Supporting information: this article has supporting information at journals.iucr.org/b

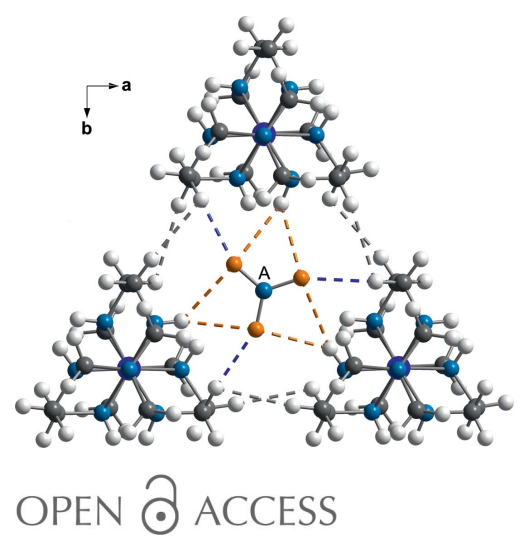

\section{The $Z^{\prime}=12$ superstructure of $\Lambda$-cobalt(III) sepulchrate trinitrate governed by $\mathrm{C}-\mathrm{H} \cdots \mathrm{O}$ hydrogen bonds}

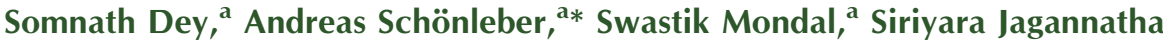 \\ Prathapa, ${ }^{a}$ Sander van Smaalen ${ }^{a}$ and Finn Krebs Larsen ${ }^{b}$
}

aLaboratory of Crystallography, University of Bayreuth, Bayreuth, Germany, and ${ }^{\mathbf{b}}$ Department of Chemistry, Aarhus
University, Aarhus, Denmark. *Correspondence e-mail: andreas.schoenleber@uni-bayreuth.de

$\Lambda$-Cobalt(III) sepulchrate trinitrate crystallizes in $P 6_{3} 22$ with $Z=2\left(Z^{\prime}=1 / 6\right)$ at room temperature. Slabs perpendicular to the hexagonal axis comprise molecules Co(sepulchrate) alternating with nitrate groups $A$ and $B$. Coordinated by six sepulchrate molecules, highly disordered nitrate groups $C$ are accommodated between the slabs. Here we report the fully ordered, lowtemperature crystal structure of $\mathrm{Co}(\mathrm{sep})\left(\mathrm{NO}_{3}\right)_{3}$. It is found to be a high- $Z^{\prime}$ structure with $Z^{\prime}=12$ of the 12 -fold $6 a_{h} \times \sqrt{3} b_{h} \times c_{h}$ superstructure with monoclinic symmetry $P 2_{1}$ (c unique). Correlations between structural parameters are effectively removed by refinements within the superspace approach. Superstructure formation is governed by a densification of the packing in conjunction with ordering of nitrate group $C$, the latter assuming different orientations for each of the $Z^{\prime}=12$ independent copies in the superstructure. The Co(sep) moiety exhibits small structural variations over its 12 independent copies, while orientations of nitrate groups $A$ and $B$ vary less than the orientations of the nitrate group $C$ do. Molecular packing in the superstructure is found to be determined by short $\mathrm{C}-\mathrm{H} \cdots \mathrm{H}-\mathrm{C}$ contacts, with $\mathrm{H} \cdots \mathrm{H}$ distances of $2.2-2.3 \AA$, and by short $\mathrm{C}-\mathrm{H} \cdots \mathrm{O}$ contacts, with $\mathrm{H} \cdots \mathrm{O}$ distances down to $2.2 \AA$. These contacts presumably represent weak $\mathrm{C}-\mathrm{H} \cdots \mathrm{O}$ hydrogen bonds, but in any case they prevent further densification of the structure and strengthening of weak $\mathrm{N}-\mathrm{H} \cdots \mathrm{O}$ hydrogen bonds with observed $\mathrm{H}$. . O distances of 2.4-2.6 ̊.

\section{Introduction}

Macrobicyclic metal cage complexes are templating agents for the synthesis of silicates (Hondow et al., 2012) and zeolites (Garcia et al., 2001). These compounds also serve as catalysts enhancing electrochemical processes (Ritzert et al., 2013), as a photosensitizer for the production of hydrogen from water (Pina et al., 1985) and as reducing agents in the field of bioelectrochemistry (Bernhardt et al., 2006). The crystal structures of these compounds provide useful information because applications of these compounds are correlated to their thermodynamic stability, stereochemistry, size and the nature of coordination inside the cages, and the strong and weak interaction of the cages with the functional groups (Gahan \& Harrowfield, 2015).

$\Lambda$-Cobalt(III) sepulchrate trinitrate, $\mathrm{Co}(\mathrm{sep})\left(\mathrm{NO}_{3}\right)_{3}$ [IUPAC name $\Lambda$ - $(1,3,6,8,10,13,16,19$-octaazabicyclo[6,6,6]eicosane)cobalt(III) trinitrate] is a sepulchrate cage complex. The sepulchrate moiety consists of a $\mathrm{Co}^{3+}$ cation sixfold coordinated by $\mathrm{N}$ atoms of the six amine groups $\left(\mathrm{N}_{\text {lig }}=\mathrm{N} 21\right.$, N22, N23, N24, N25, N26 in Fig. 1) of the sepulchrate molecule. The amine groups act as donors of lone pairs of electrons 
to the $d^{2} s p^{3}$ hybridized $\mathrm{Co}^{3+}$ cation conforming a distorted octahedron. The macrobicyclic cage is completed by three ethylene groups $\left(\mathrm{C}_{\mathrm{en}}=\mathrm{C} 31, \mathrm{C} 36\right.$; C33, C34; C32, C35), six apical $\mathrm{C}$ atoms $\left(\mathrm{C}_{\mathrm{ap}}=\mathrm{C} 41, \mathrm{C} 42, \mathrm{C} 43, \mathrm{C} 44, \mathrm{C} 45, \mathrm{C} 46\right)$ and two apical $\mathrm{N}$ atoms $\left(\mathrm{N}_{\mathrm{ap}}=\mathrm{N} 51, \mathrm{~N} 52\right.$ in Fig. 1$)$.

The compound crystallizes in hexagonal symmetry with space group $\mathrm{P6}_{3} 22$ at room temperature (phase I) (Dubicki $e t$ al., 1980; Schönleber et al., 2010). The Co(sep) cage and two symmetry-related nitrate groups (nitrate groups $A$ and $B$ ) are centred on threefold rotation axes. They have been reported to be connected via $\mathrm{N}-\mathrm{H} \cdots \mathrm{O}$ hydrogen bonds (Dubiciki et al., 1984). The third nitrate group (nitrate group $C$ ) is slightly displaced from the origin, exhibiting a sixfold orientational disorder (Schönleber et al., 2010).

A phase transition of $\mathrm{Co}(\mathrm{sep})\left(\mathrm{NO}_{3}\right)_{3}$ has been found to occur at $T_{1}=133 \mathrm{~K}$; spectroscopic methods have indicated that below $T_{1}$ the crystals develop a domain structure, as it may appear due to a lowering of its symmetry (Dubiciki et al., 1984). Single-crystal neutron diffraction experiments have revealed satellite reflections below $T_{1}$. Their temperature dependence has indicated two further phase transitions, at $T_{2}=107 \mathrm{~K}$ and $T_{3}=98 \mathrm{~K}$, respectively (Larsen et al., 1988).

We have performed temperature-dependent single-crystal $\mathrm{X}$-ray diffraction studies on $\mathrm{Co}(\mathrm{sep})\left(\mathrm{NO}_{3}\right)_{3}$, which confirmed the existence of different phases at $T=115 \mathrm{~K}$ (phase II), $100 \mathrm{~K}$ (phase III) and $95 \mathrm{~K}$ (phase IV; Dey et al., 2016). The two intermediate phases II and III possess incommensurately modulated crystal structures (Larsen et al., 1988).

The present X-ray diffraction experiments reveal the lowtemperature phase (phase IV) to be a lock-in phase with a 12fold $6 a_{h} \times \sqrt{3} b_{h} \times c_{h}$ superstructure of the room-temperature hexagonal structure. The phase transition from phase III to

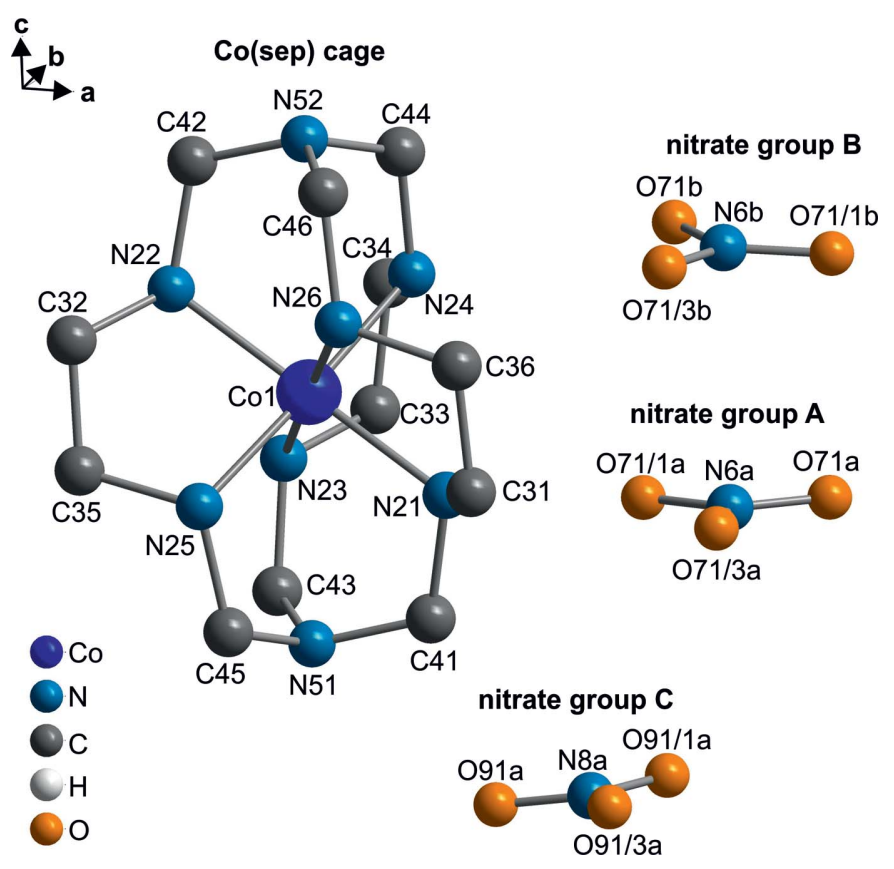

Figure 1

Molecular structure of of $\Lambda$-cobalt(III) sepulchrate trinitrate along with the atomic numbering scheme. $\mathrm{H}$ atoms have been omitted for clarity phase IV is identified as an incommensurate-to-commensurate transition accompanied by major changes of the modulation wave. Specifically, the disordered nitrate group $C$ becomes fully ordered in phase IV, assuming different orientations at different sites within the 12-fold supercell.

The order as well as the accompanying atomic displacements and molecular distortions are described by modulation functions within the superspace approach (van Smaalen, 2012; Schönleber, 2011; Janssen et al., 2007).

The origin of the modulation is discussed in terms of molecular conformations and intermolecular interactions, including the role of hydrogen bonding.

\section{Experimental}

\subsection{Diffraction experiment and data integration}

Single crystals of $\mathrm{Co}(\mathrm{sep})\left(\mathrm{NO}_{3}\right)_{3}$ were synthesized by the research team of Alan M. Sargeson (Creaser et al., 1982). The crystals are stable in air at ambient conditions. Single-crystal $\mathrm{X}$-ray diffraction experiments were performed at beamline D3, Hasylab DESY, Hamburg, employing radiation of a wavelength of $\lambda=0.50917 \AA$. The sample was mounted on a four-circle diffractometer in Euler geometry, and diffraction intensities were measured by a MAR-CCD area detector. A data set complete up to a resolution of $(\sin \theta / \lambda)_{\max }=$ $0.838 \AA^{-1}$ was measured by $\phi$ and $\omega$ scans with several exposure times and different offsets of the detector, always maintaining a crystal-to-detector distance of $220 \mathrm{~mm}$ and using scans of $1^{\circ}$ per image (Table 1 ).

Data processing has been performed with the software EVAL15 (Schreurs et al., 2010). Inspection of the frames revealed that main reflections are surrounded by satellite reflections of first order. Bragg reflections have been indexed using five integers $\left(h k l m_{1} m_{2}\right)$ on a primitive hexagonal lattice and with modulation wavevectors

$$
\begin{aligned}
\mathbf{q}^{1} & =\left(\sigma_{h}, \sigma_{h}, 0\right) \\
\mathbf{q}^{2} & =\left(-2 \sigma_{h}, \sigma_{h}, 0\right) .
\end{aligned}
$$

Refinement of the hexagonal orientation matrix and the component of the modulation wavevector resulted in $2 \sigma_{h}=$ $0.16726(10)=\frac{1}{6}+0.00059(10)$, suggesting an incommensurate modulation.

However, diffraction images exhibit diffraction maxima that appear as overlapping Bragg reflections from different domains rather than single peaks. Therefore, we have used an alternative indexing of the diffraction pattern on the $C$ centred $a_{h} \times \sqrt{3} b_{h} \times c_{h}$ orthohexagonal unit cell. In the case of orthorhombic symmetry, each main reflection is composed of three different, nearly coincident Bragg reflections from the three domains, while satellite reflections have contributions from only a single domain (Fig. 2a). With respect to the orthohexagonal unit cell the satellite reflections are indexed by $\mathbf{q}^{\mathrm{o}}=\left(\sigma_{\mathrm{o}}, 0,0\right)$ with $\sigma_{\mathrm{o}} \simeq 2 \sigma_{h}$.

In the case of monoclinic symmetry, each main reflection is composed of six different, nearly coincident Bragg reflections from the six domains; satellite reflections now have contri- 
Table 1

Experimental and crystallographic data.

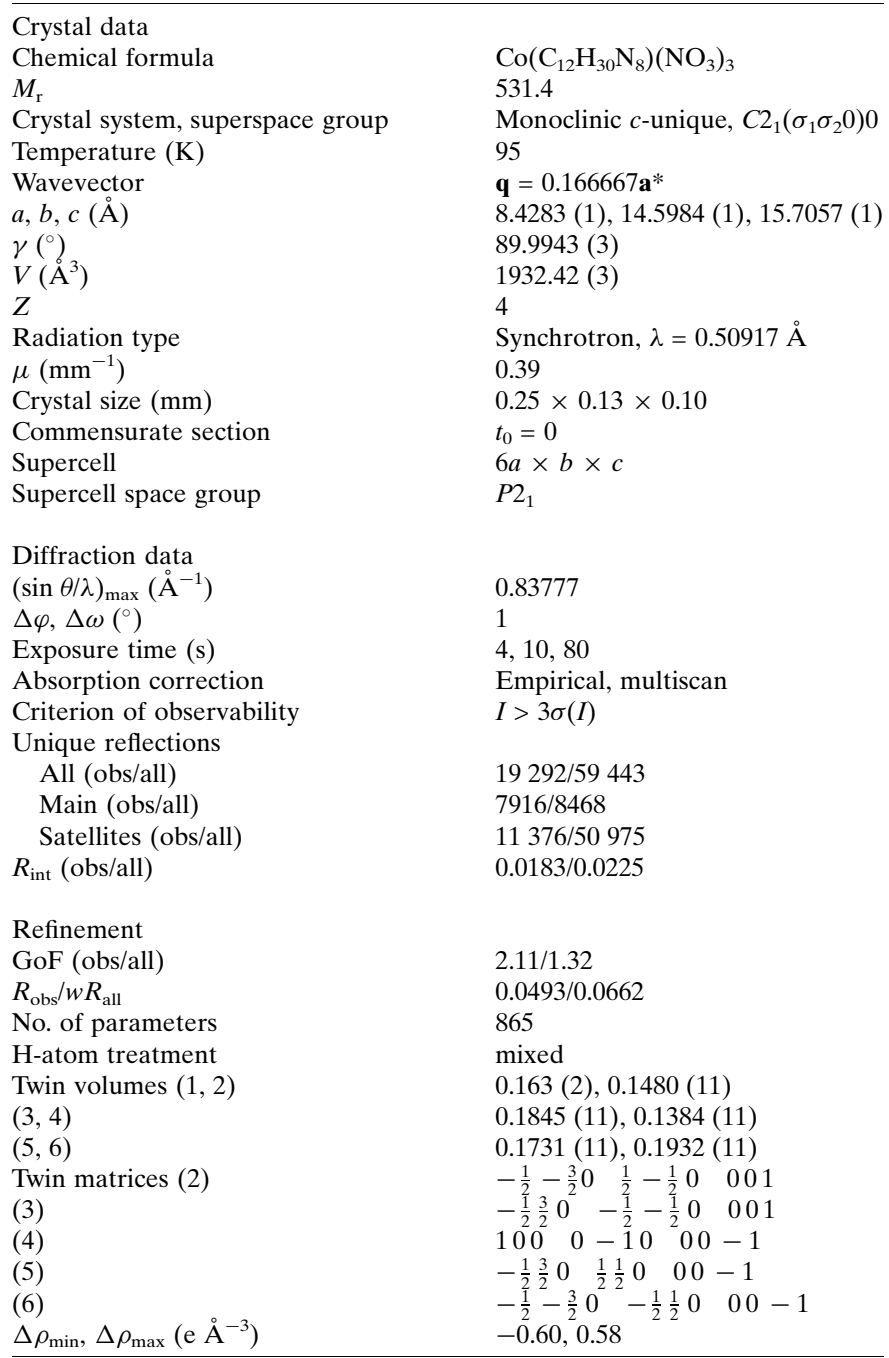

butions from two domains (Fig. 2). Each domain of monoclinic symmetry is modulated by a single modulation wavevector

$$
\mathbf{q}=\left(\sigma_{\mathrm{m}}, \sigma_{2}, 0\right)
$$

with $\sigma_{\mathrm{m}} \simeq 2 \sigma_{h}$ and $\sigma_{2} \simeq 0$.

Refinement with EVAL15 of the orthorhombic lattice parameters and the orientations of the six lattices resulted in a very small orthorhombic lattice distortion together with $\sigma_{\mathrm{o}}=\frac{1}{6}-0.000071$ (26). Further lowering of the symmetry to monoclinic resulted in a lattice distortion given by the angle $\gamma=89.9943(3)^{\circ}$ and $\sigma_{\mathrm{m}}=\frac{1}{6}-0.000026$ (9). This value is much closer to the commensurate value $\frac{1}{6}$ than the value obtained with the hexagonal lattice and it differs from $\frac{1}{6}$ by less than three standard uncertainties. The discrepancies can be explained by the fact that the twinned refinement determines the position of the modulation wavevector with respect to the reciprocal lattice in its own domain, whereas the two-dimensional modulation on the hexagonal lattice determines the average satellite position with respect to the average lattice of all domains. A non-zero value for $\sigma_{2}$ leads to instable refine- ments and a non-significant value for this parameter. Together, we take these refinements as experimental evidence for the commensurability of the modulation in phase IV.

The severe overlap of Bragg reflections from different domains prevents the determination of integrated intensities of individual reflections. Instead, accurate intensities can be obtained for each group of overlapping reflections. Furthermore, available software for absorption correction cannot simultaneously handle twinned and incommensurate data. Therefore, integrated intensities have been determined within the hexagonal setting, employing the two modulation wavevectors from equation (1). Integrated intensities have been obtained for main reflections, $(h k l 00)$ and satellite reflections of first order, $(h k l \pm 10),(h k l 0 \pm 1)$ and $(h k l \pm 1 \mp 1)$, employing EVAL15. The absorption correction was determined by $S A D A B S$ (Sheldrick, 2008). Throughout these calculations, monoclinic point symmetry $2 / m$ (c unique) has been used. This is the minimum symmetry of the diffraction pattern in the case of twin domains of unequal volume fractions. Only for equal volume fractions of the domains can the
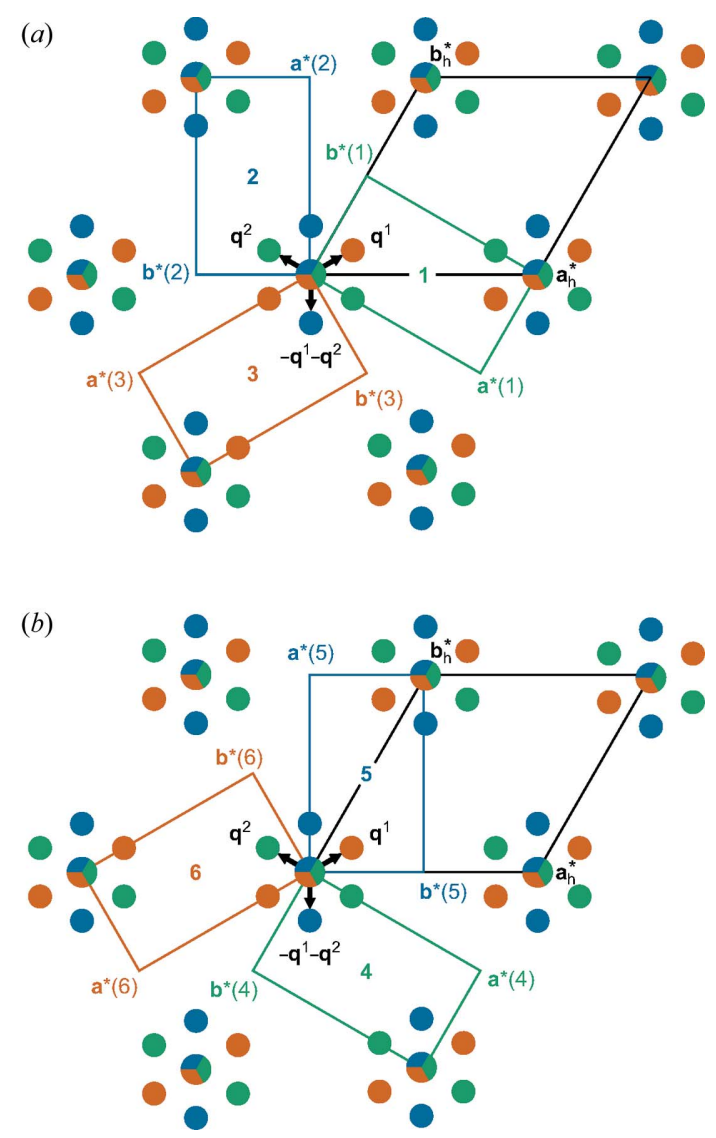

Figure 2

Schematic drawing of the diffraction pattern at $T=95 \mathrm{~K}$ exhibiting hexagonal to monoclinic sixfold twinning. (a) $\mathbf{q}^{2}, \mathbf{q}^{1}+\mathbf{q}^{2}$ and $\mathbf{q}^{1}$ belong to the green (1), blue (2) and red (3) orthorhombic or monoclinic domains, respectively. (b) Monoclinic twin domains 4 , and 5 and 6 are generated by applying twofold rotation a axes parallel to the twin domains 1,2 and 3, respectively. The assignment of the q-vectors to the twin domains has been chosen consistent with International Tables for Crystallography, Vols. A and C (Arnold, 2006; Janssen et al., 2006). The reconstructed reciprocal layer $h k 2$ is given as Fig. S1 in the supporting information. 
point symmetry become higher, e.g. $6 / \mathrm{mmm}$ in the case of all volume fractions being equal.

After data reduction, the dataset was split into one subset of main reflections (common to all twin domains) and three subsets of satellites reflections (for the three twin domains 1,2 and 3; Fig. $2 a$; twin matrices are given in Table 1). The indices were transformed from $\left(h \mathrm{klm} \mathrm{m}_{1} \mathrm{~m}_{2}\right)$ to $(\mathrm{hklm})$ without changing $h, k$ or $l$, and with $m=-m_{2}$ for the twin domain 1, $m=m_{1}+m_{2}$ for twin domain 2 and $m=-m_{1}$ for twin domain 3 . The reflection indices were then transformed to the $C$-centred orthohexagonal setting (Arnold, 2006), using the software JANA2006 (Petricek et al., 2014). The three additional monoclinic twin domains 4,5 and 6 were described by applying a twofold rotation along a to the domains 1,2 and 3, respectively. In this way, satellite reflections of each of the three pairs of domains coincide with each other according to: ( $h k l m)$ of domain 1 coincides with $(h-k-l m)$ of domain 4; main reflections of all six domains coincide. The ratio of the average intensities $\langle I\rangle$ between main and satellite reflections is approximately $60: 1$, while the average significance $\langle I / \sigma(I)\rangle$ has a ratio of $4: 1$ between the main and satellite reflections.

\subsection{Structure solution and refinement}

A model for the 12-fold superstructure of phase IV of $\mathrm{Co}(\mathrm{sep})\left(\mathrm{NO}_{3}\right)_{3}$ at $T=95 \mathrm{~K}$ has been developed within the superspace approach (van Smaalen, 2012). The basic structure is nearly identical to the crystal structure of phase I, but it has been described on the $C$-centred orthohexagonal unit cell (Table 1). Consideration of diffraction symmetry (Table 1 ) and reflection conditions has led to the space group $C 2_{1}$ (c-unique) for the basic structure, where the monoclinic unique axis is equal to the hexagonal axis of phase I. This space group is a non-standard setting of $P 2_{1}$, No. 4 in International Tables for Crystallography, Vol. A. The superspace group has been found as $C 2_{1}\left(\sigma_{1} \sigma_{2} 0\right) 0$ with $\sigma_{1}=\sigma_{m}=\frac{1}{6}$ and $\sigma_{2}=0$. This superspace group is a non-standard setting of $P 2_{1}\left(\sigma_{1} \sigma_{2} 0\right) 0$, No. 4.1.2.1 in Stokes et al. (2011) and van Smaalen et al. (2013).

Formally, charge flipping does not apply to diffraction data from crystals twinned by (pseudo-)merohedry. Nevertheless, SUPERFLIP (Palatinus \& Chapuis, 2007) applied to the present data allowed us to determine the positions of the Co atom along with initial values for its modulation parameters. The positions of the other non- $\mathrm{H}$ atoms were derived from the atomic positions of phase I (Schönleber et al., 2010), employing JANA2006 for the application of group-subgroup relations following the reduction of symmetry from $\mathrm{Pb}_{3} 22$ to $C 222_{1}$ to $C 2_{1}$. The low monoclinic symmetry of the pseudohexagonal basic structure is responsible for large correlations between structural parameters. Therefore, restraints on interatomic distances and bond angles of the Co(sep) cage have been introduced in accordance with the crystal structure of phase I (Schönleber et al., 2010). The nitrate groups have been described as planar molecules with point symmetry 32 , leaving the $\mathrm{N}-\mathrm{O}$ distance as a single refinable parameter. One nitrate molecule is placed in two independent positions corresponding to the nitrate groups $A$ and $B$. A second nitrate molecule is used for nitrate group $C$. Each independent position is defined by three translational and three rotational parameters. The modulations of the nitrate groups were initially described by small values for the amplitudes of the first-order harmonic waves for modulation of translations and rotations. Displacement modulations of the other non- $\mathrm{H}$ atoms have been described by a single harmonic wave with initially small values for the parameters, except cobalt, for which the parameters obtained by SUPERFLIP have been used. $\mathrm{H}$ atoms were added to all ligand $\mathrm{N}$ atoms $\left(\mathrm{N}_{\mathrm{lig}}\right)$, and all ethylene $\left(\mathrm{C}_{\mathrm{en}}\right)$ and apical $\left(\mathrm{C}_{\mathrm{ap}}\right) \mathrm{C}$ atoms by a riding model, keeping tetrahedral geometries with distances $d(\mathrm{~N}-\mathrm{H})=$ $0.87 \AA$ and $d(\mathrm{C}-\mathrm{H})=0.96 \AA$. Atomic displacement parameters (ADPs) of $\mathrm{H}$ atoms follow from $U_{\text {iso }}(\mathrm{H})=1.2$ $U_{\text {eq }}$ (parent atom). Structure refinements of this $(3+1)$ dimensional superspace model have been performed with $J A N A 2006$. Employing isotropic ADPs and the incommensurate approximation, the refinement converged at $R_{F}^{\mathrm{obs}}($ main $)=0.1254$ and $R_{F}^{\mathrm{obs}}(\mathrm{sat})=0.1564$ for main and satellite reflections, respectively.

In the next step, anisotropic ADPs were introduced for the non-H atoms in the $\mathrm{Co}(\mathrm{sep})$ cage and TLS parameters (Schomaker \& Trueblood, 1968) were applied to the nitrate groups. Refinement resulted in a much improved fit to the main reflections with $R_{F}^{\text {obs }}$ (main) $=0.0425$ and $R_{F}^{\mathrm{obs}}(\mathrm{sat})=0.1451$, and a large reduction of the features in the difference Fourier map, viz $\Delta \rho_{\min } / \Delta \rho_{\max }$ reduced from $-2.26 /$ 10.84 to $-1.02 / 2.19 \mathrm{e}^{-3}$. Subsequently, first-order harmonic waves have been introduced for the modulation of ADPs of non- $\mathrm{H}$ atoms of the Co(sep) cage and for modulation of the TLS parameters of the nitrate groups. Refinement resulted in an improved fit to the satellite reflections with $R_{F}^{\mathrm{obs}}($ main $)=0.0389$ and $R_{F}^{\mathrm{obs}}(\mathrm{sat})=0.1052$.

The next step involved the introduction of first-order harmonic waves for the third-order Gram-Charlier parameters of the Co atom, while the basic third-order GramCharlier parameters were constrained to remain zero (Li et al., 2011). The fit to the diffraction data improved again at $R_{F}^{\text {obs }}($ main $)=0.0376$ and $R_{F}^{\text {obs }}(\mathrm{sat})=0.1002$. More importantly, ADPs of the ten atoms in the Co(sep) cage, which were previously non-positive definite, become positive definite by this procedure.

In the final model constraints of the riding model on the fractional coordinates and modulation parameters of the $\mathrm{H}$ atoms attached to the six $\mathrm{N}_{\text {lig }}$ atoms were replaced by restraints on distances and angles. Restraint parameters are $d(\mathrm{~N}-\mathrm{H})=0.87 \pm 0.02 \AA$ and $\angle(\mathrm{H}-\mathrm{N}-\mathrm{Co})=\angle(\mathrm{H}-\mathrm{N}-$ $\mathrm{C} 3)=\angle(\mathrm{H}-\mathrm{N}-\mathrm{C} 4)=109.47 \pm 1^{\circ}$. Refinement resulted in an again improved fit, with $R_{F}^{\text {obs }}($ main $)=0.0373$ and $R_{F}^{\text {obs }}($ sat $)=0.0998$, and much reduced features in the difference Fourier map (Table 1 ). The plot of $F_{\text {obs }}$ versus $F_{\text {calc }}$ shows an excellent match between these quantities (Fig. S2 in the supporting information).

The commensurability of the modulation requires the selection of the correct value of the initial phase, $t_{0}$, of the modulation wave (van Smaalen, 2012). For the 12-fold superstructure, the modulation wave is sampled at 12 equidi- 
Table 2

Statistical parameters $\left(R_{F}^{\text {obs }}\right.$ and $\left.w R_{F^{2}}^{\text {all }}\right)$ of commensurate refinements of the final model with different values of the phase $t_{0}$.

\begin{tabular}{|c|c|c|c|}
\hline & $t_{0}=0$ & $t_{0}=\frac{1}{48}$ & $t_{0}=\frac{1}{24}$ \\
\hline Supercell space group & $P 2_{1}$ & $P 1$ & $P 2_{1}$ \\
\hline$R_{F}^{\mathrm{obs}}($ all $)$ & 0.0493 & 0.0496 & 0.0493 \\
\hline$w R_{F^{2}}^{\text {all }}$ (all) & 0.0662 & 0.0665 & 0.0662 \\
\hline$R_{F}^{\text {obs }}($ main $)$ & 0.0374 & 0.0378 & 0.0374 \\
\hline$w R_{F^{2}}^{\text {all }}$ (main) & 0.0490 & 0.0496 & 0.0490 \\
\hline$R_{F}^{\text {obs }}(\mathrm{sat})$ & 0.0998 & 0.1000 & 0.0998 \\
\hline$w R_{F^{2}}^{\text {all }}$ (sat) & 0.1250 & 0.1252 & 0.1250 \\
\hline$\Delta \rho_{\min }\left(\mathrm{e} \AA^{-3}\right)$ & -0.58 & -0.61 & -0.58 \\
\hline$\Delta \rho_{\max }\left(\mathrm{e} \AA^{-3}\right)$ & 0.60 & 0.59 & 0.60 \\
\hline
\end{tabular}

stant points. Since only first-order satellite reflections have been observed (Fig. 3), this implies that different $t_{0}$ as well as the incommensurate model provide fits to the diffraction data of nearly equal quality. Table 2 gives partial $R$ values for three different values of $t_{0}$. We believe that the minor differences in $R$ values are not sufficient to uniquely determine $t_{0}$. Nevertheless, $t_{0}=\frac{1}{48}$ has slightly higher $R$ values and represents a supercell structure with triclinic symmetry, while $t_{0}=0$ gives the lowest $R$ values and corresponds to the highest supercell symmetry with space group $P 2_{1}$ (a model with the same symmetry is obtained for $\left.t_{0}=\frac{1}{24}\right)$. Evidence for lowering the symmetry to triclinic thus is lacking and we have chosen the monoclinic model with $t_{0}=0$ for further analysis.

The relatively high partial $R_{F}^{\text {obs }}$ (sat) value for satellite reflections (Table 2) can be explained by rather weak reflections, as it is expressed by the magnitudes of partial $R$ values for satellite reflections for the averaged standard uncertainty over intensity $R_{\sigma}$ (sat) $=0.1510$ and for averaging of equivalent reflections $R_{\text {int }}$ (sat) $=0.1420$. Further evidence for the final structure model has been obtained by establishing that

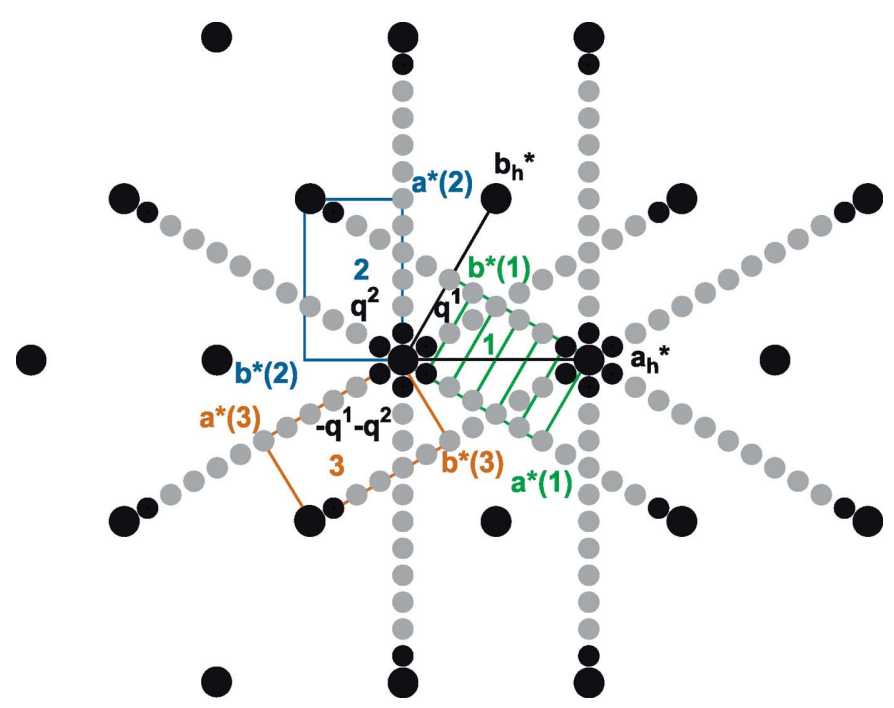

Figure 3

Schematic drawing of the diffraction pattern at $T=95 \mathrm{~K}$. Positions are indicated of all possible commensurate satellite reflections. Black circles represent observed reflections while grey circles denote reflection positions unobserved in the present experiment. The sixfold supercell corresponding to the green domain in Fig. 2 is highlighted by thin lines. the following variations of the model did not improve the fit to the diffraction data:

(i) Treatment of nitrate group $A$ and nitrate group $B$ as different molecules leads to large correlations in the positions of their $\mathrm{O}$ atoms without any significant changes in $R$ values.

(ii) The Co(sep) cage was refined without distance and angle restraints and individual atoms were refined for the nitrate groups. This model results in improved statistical parameters of refinement $\left[R_{F}^{\text {obs }}(\right.$ main $)=0.0335$ and $R_{F}^{\text {obs }}($ sat $)=$ 0.0975], but the ADPs of four C atoms are non-positive definite and the geometries of the nitrate groups are outside the physically possible range.

(iii) Since the symmetry is non-centrosymmetric, inversion twinning was introduced for all six twin domains. The refinement resulted in insignificant values of the inversion twin volumes.

(iv) Second-order harmonic waves were added for displacive modulation of the atoms, but the resulting modulation functions showed ripples indicating fitting of the noise in the data.

(v) Isotropic secondary extinction correction resulted in a negative extinction parameter.

\section{Discussion}

\subsection{Variation of molecular conformations for $Z^{\prime}=12$}

The crystal structure of the low-temperature phase (phase IV) of $\mathrm{Co}(\mathrm{sep})\left(\mathrm{NO}_{3}\right)_{3}$ is a high- $Z^{\prime}$ structure. Only a few crystal structures feature more independent molecules in the unit cell than the presently observed $Z^{\prime}=12$ [for example, the amino acid L-tryptophan with $Z^{\prime}=16$ (Görbitz et al., 2012); see also the review article by Steed \& Steed (2015) and references therein, as well as the high $Z^{\prime}$ structure database by Steed (2016)]. High- $Z^{\prime}$ structures usually suffer from severe correlations between parameters in structure refinements, and restrictions on the parameters beyond symmetry restrictions are necessary. An elegant way of imposing nonsymmetry restrictions is the superspace approach (Schönleber, 2011; van Smaalen, 2012; Pinheiro \& Abakumov, 2015). Parameters are separated into a relatively small subset of atomic coordinates describing the basic structure [one molecule $\mathrm{Co}(\mathrm{sep})$ and three nitrate ions] and parameters describing deviations from the basic structure. For $\mathrm{Co}(\mathrm{sep})\left(\mathrm{NO}_{3}\right)_{3}$ we have observed satellite reflections in the X-ray diffraction of first order only (Fig. 3), which implies that the superstructure is described by a single harmonic modulation function for each independent parameter of the basic structure; higher harmonics cannot be determined (see the end of \$2.2). Deviations from the basic structure are small indeed, as is easily seen by consideration of a plot of the superstructure, showing the 12 crystallographically independent but nearly indistinguishable copies of the molecule (Figs. 4 and 5).

The superspace approach reduces the number of independent parameters dramatically compared with the 12 -fold supercell. Nevertheless, the monoclinic superspace group of phase IV does not capture the pseudo-trigonal symmetry of 


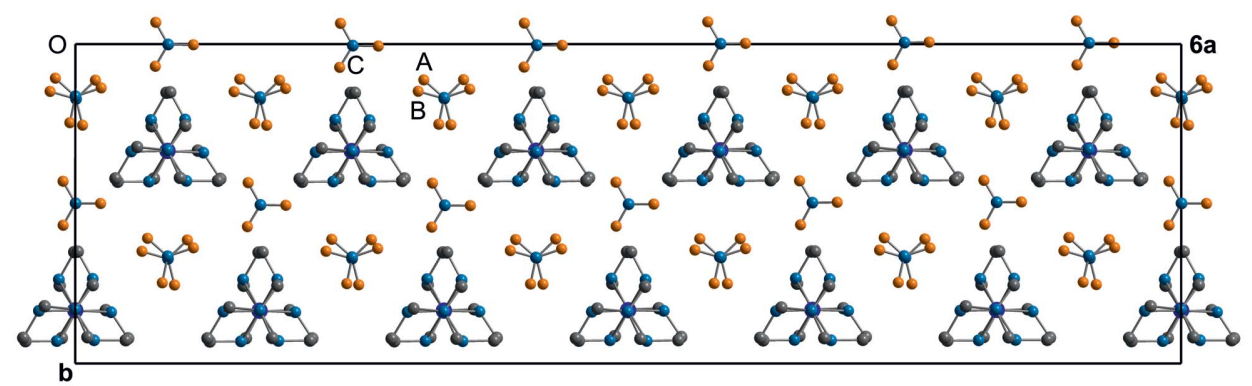

Figure 4

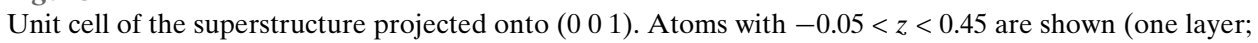
$c f$. Fig. 5). $\mathrm{H}$ atoms are not shown.

the Co(sep) molecule, and further restrictions have turned out to be necessary. We have chosen to use rather narrow restraints on bond lengths and bond angles $(\$ 2.2)$. They are justified in hindsight by the good fit to the diffraction data of the thus refined structure model (Tables 1 and 2).

Unsurprisingly, the 12 crystallographically independent Co(sep) molecules in the 12-fold supercell feature similar values for each bond length and each bond angle. The variations are most favourably analysed by $t$-plots (van Smaalen,
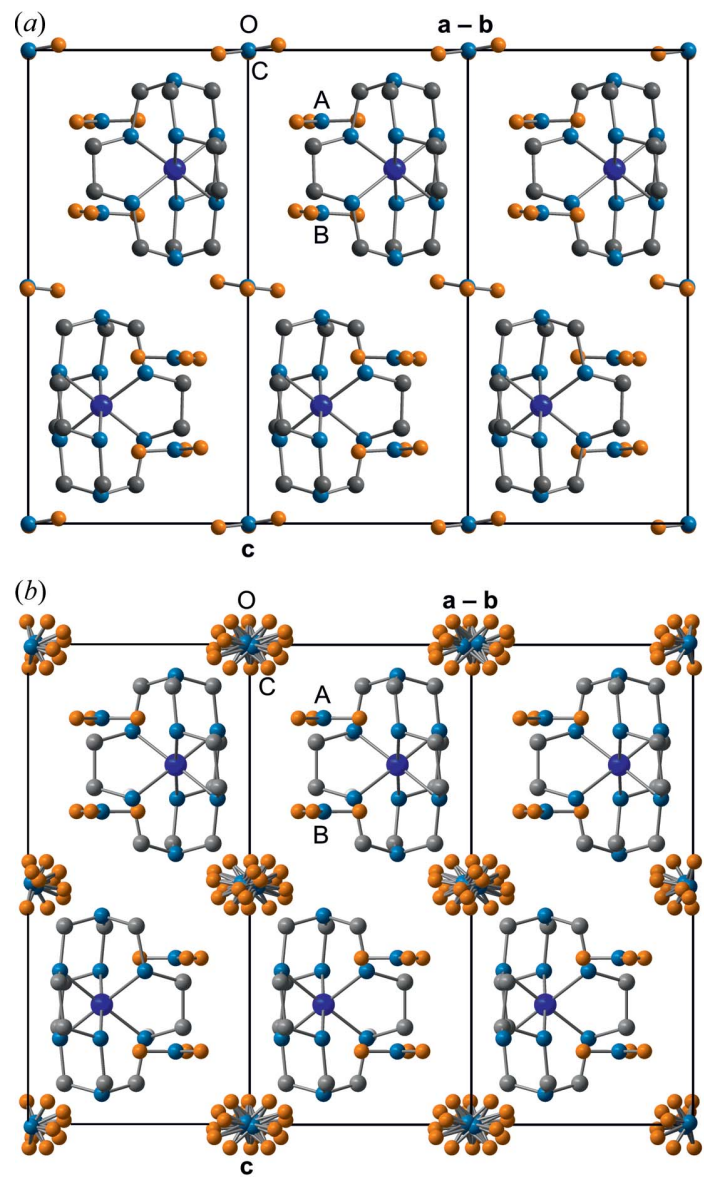

Figure 5

(a) The basic structure at $T=95 \mathrm{~K}$ (phase IV) projected onto (1 110$)$ and (b) the disordered phase at room temperature (phase I; Schönleber et al., 2010). $\mathrm{H}$ atoms are not shown.
2012), which provide the continuous dependence of each structural parameter on phase $t$ of the modulation wave. Values observed in the 12-fold superstructure with $t_{0}=0$ are the values $t=n / 12$ for $n=$ $0, \ldots, 11$. Fig. 6 provides a $t$-plot of the distances between the Co atom and the $12 \mathrm{C}$ atoms of the same molecule. It shows that these distances (not restrained in the refinement) have but minor variations between the 12 molecules. The (small) elongation of one $\mathrm{Co}-$ $\mathrm{C}$ bond is compensated for by shortening another $\mathrm{Co}-\mathrm{C}$ bond, in complete agreement with the behaviour of other modulated compounds. Presently, distances towards the three apical $\mathrm{C}$ atoms at the top of the molecule (Nos. 10-12 in Fig. 6) are longer than average simultaneously with the distances towards the three apical C atoms at the bottom of the molecule being shorter than average (Nos. 7-9). This reflects the largest modulation amplitude of Co being along $\mathbf{c}$ (Table S1 in the supporting information). Angles within the $\mathrm{Co}\left(\mathrm{N}_{\mathrm{lig}}\right)_{6}$ octahedron deviate more from their ideal values than in the structure of phase I (Schönleber et al., 2010), but their variation with $t$ is again very small (see the supporting information). Bond lengths and bond angles of all 12 independent $\mathrm{Co}(\mathrm{sep})$ molecules thus are similar to each other. The average molecule in phase IV has a slightly more distorted geometry than the structure of the molecule within the high-symmetry phase (Schönleber et al., 2010) as well as in other filled sepulchrate complexes (Bacchi et al., 1993; Bacchi et al., 1996; Gahan \& Harrowfield, 2015).

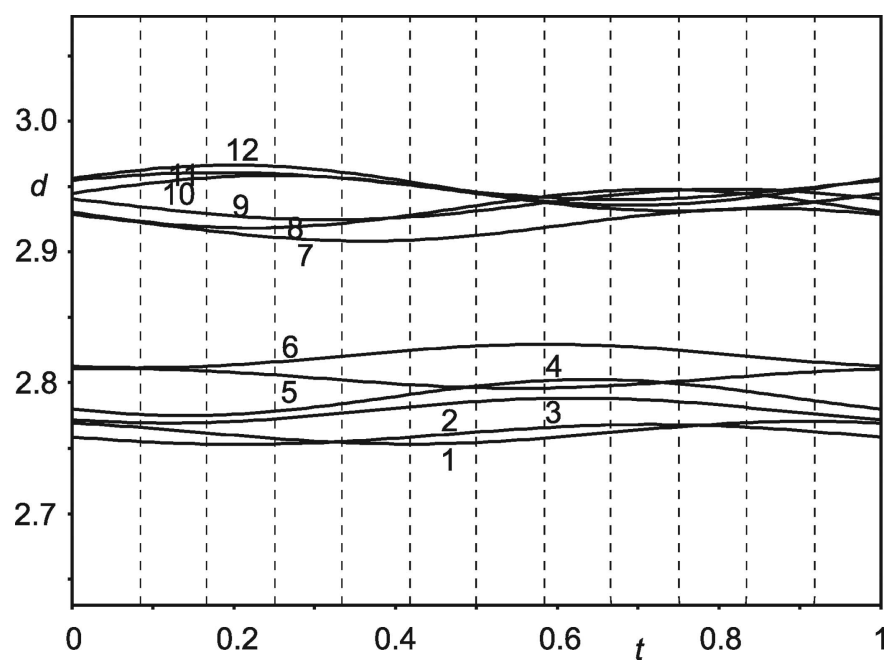

Figure 6

$t$-Plot of interatomic distances $d(\AA)$ between the Co atom and the $\mathrm{C}$ atoms of the same molecule. Vertical, dashed lines indicate $t$ values corresponding to the distances in the 12-fold supercell. The 12 interatomic contacts are (cf. Fig. 1$): 1=\mathrm{Co} 1-\mathrm{C} 36,2=\mathrm{Co} 1-\mathrm{C} 34,3=$ $\mathrm{Co} 1-\mathrm{C} 33,4=\mathrm{Co} 1-\mathrm{C} 35,5=\mathrm{Co} 1-\mathrm{C} 31,6=\mathrm{Co} 1-\mathrm{C} 32,7=\mathrm{Co} 1-\mathrm{C} 45,8$ $=\mathrm{Co} 1-\mathrm{C} 43,9=\mathrm{Co} 1-\mathrm{C} 41,10=\mathrm{Co} 1-\mathrm{C} 44,11=\mathrm{Co} 1-\mathrm{C} 42$ and $12=$ Co1-C46. 


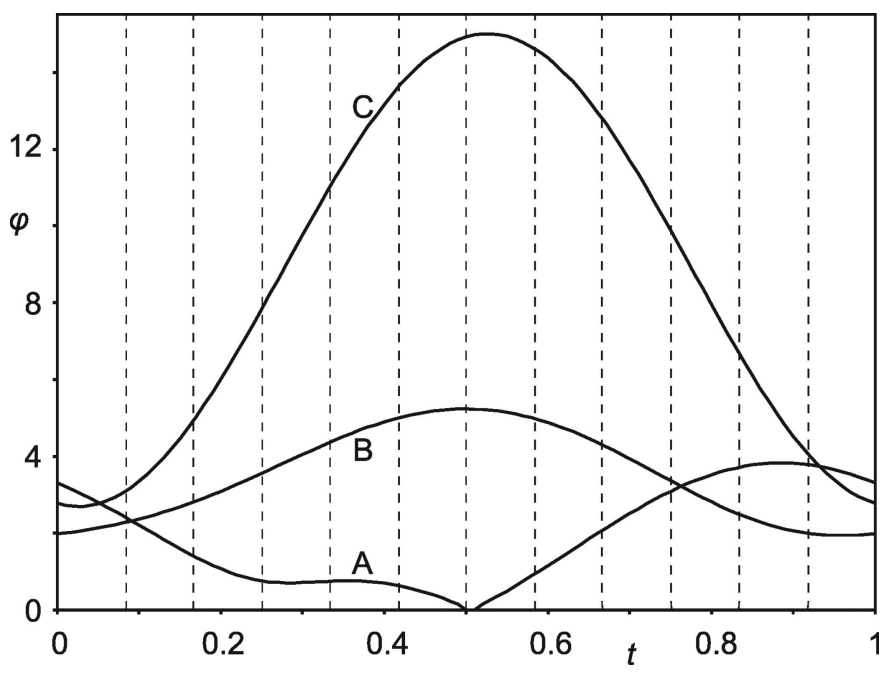

Figure 7

$t$-Plot of the angles $\varphi\left({ }^{\circ}\right)$ between the $(\mathbf{a}, \mathbf{b})$-plane and the molecular planes of the nitrate groups $A, B$ and $C$. Vertical dashed lines indicate $t$ values corresponding to the angles in the 12 -fold supercell.

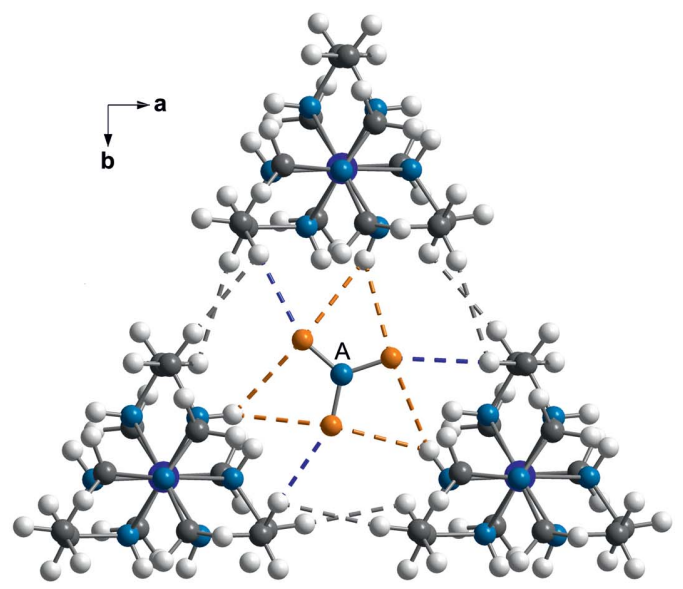

(a)

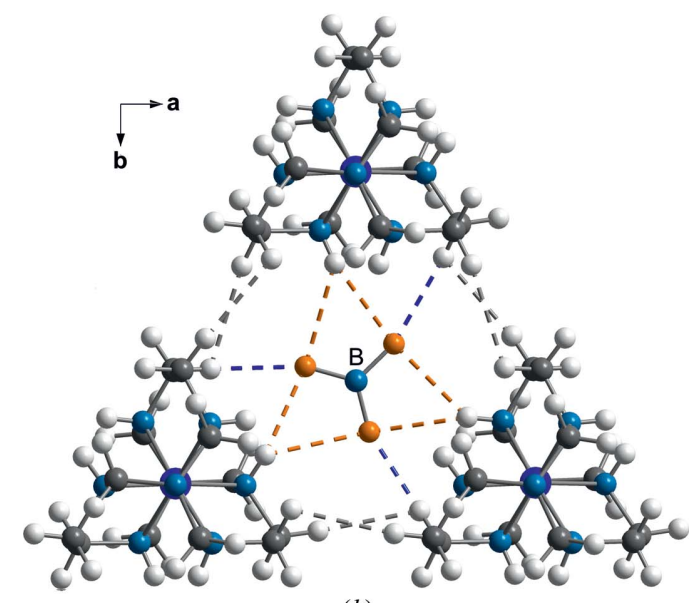

(b)

Figure 8

Environments of the nitrate groups $A$ and $B$ in a view along $\left(\begin{array}{lll}0 & 0 & 1\end{array}\right)$. Dashed lines indicate $\mathrm{N}-\mathrm{H} \cdots \mathrm{O}$ hydrogen bonds (orange), $\mathrm{C}-\mathrm{H} \cdots \mathrm{O}$ hydrogen bonds (blue) and short $\mathrm{C}-\mathrm{H} \cdots \mathrm{H}-\mathrm{C}$ contacts between participating sepulchrate cages (grey). (a) Nitrate group $A$ at $z=0.15$; (b) nitrate group $B$ at $z=0.34$. Co(sep) cages are centred at $z=1 / 4$ (cf. Fig. 5).
Nitrate groups have been restricted to be flat with $\mathrm{O}-\mathrm{N}-$ $\mathrm{O}$ angles of $120^{\circ}$ and three equal $\mathrm{N}-\mathrm{O}$ bond lengths (Fig. 1). Nitrate groups $A$ and $B$ have been described by a common molecule with a refined $\mathrm{N}-\mathrm{O}$ bond length of 1.237 (6) $\AA$. The $\mathrm{N}-\mathrm{O}$ bond in nitrate group $C$ is shorter with $d(\mathrm{~N}-\mathrm{O})=$ 1.160 (7) A. Major differences between the different copies of the nitrate groups $A, B$ and $C$ are the orientations of these planar molecular anions (Fig. 7). We believe this part of the modulation to be at the origin of the complicated superstructure, as described in the next section.

\subsection{Competing intermolecular interactions}

In phase I, the nitrate groups $A$ and $B$ are parallel to the (a, b)-plane. In the present structure (phase IV), the planes of the 12 nitrate groups $A$ and the 12 nitrate groups $B$ make different angles with the $(\mathbf{a}, \mathbf{b})$-plane, with values between 0 and $5^{\circ}$ (Fig. 7). These small rotations are the result of optimizing the interactions between nitrate and sepulchrate moieties (see below). Nitrate group $C$ is highly disordered in phase I (Fig. $5 b$; Schönleber et al., 2010). The increased packing density at lower temperatures forces each nitrate group $C$ into a single orientation. Orientations of the 12 crystallographically independent nitrate groups $C$ exhibit a much larger variation than orientations of nitrate groups $A$ and $B$ (Fig. 7), again apparently the result of optimizing intermolecular interactions.

Nitrate groups $A$ and $B$ form $\mathrm{N}-\mathrm{H} \cdots \mathrm{O}$ hydrogen bonds with the sepulchrate cages (Fig. 8). Distances $\mathrm{H} \cdots \mathrm{O}$ vary between $\sim 2.4$ and $2.6 \AA$ for the six $\mathrm{H} \cdots \mathrm{O}$ interactions about each nitrate group, but each contact exhibits but a small variation between the 12 independent nitrate groups $A$ and $B$, as shown by the $t$-plot of these distances (4-9 in Fig. 9). This

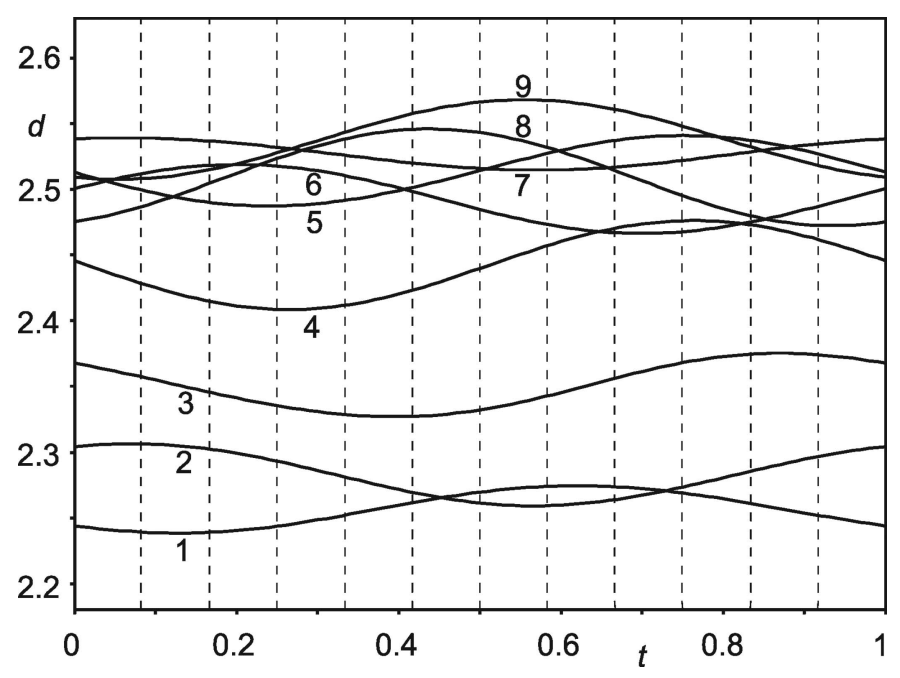

Figure 9

$t$-Plot of interatomic distances $d(\AA)$ between the $\mathrm{O}$ atom of nitrate group $A$ and $\mathrm{H}$ atoms of the sepulchate cage. Vertical, dashed lines indicate $t$ values corresponding to the distances in the 12 -fold supercell. Six N$\mathrm{H} \cdots \mathrm{O}$ contacts (4-9) and three $\mathrm{C}-\mathrm{H} \cdots \mathrm{O}$ contacts $(1-3)$ are shown $(c f$. Fig. 1): $1=\mathrm{O} 71 / 3 a \cdots \mathrm{H} 2 c 35,2=\mathrm{O} 71 a \cdots \mathrm{H} 2 c 31,3=\mathrm{O} 71 / 1 a \cdots \mathrm{H} 2 c 33,4=$ $\mathrm{O} 71 / 3 a \cdots \mathrm{H} 1 n 21,5=\mathrm{O} 71 / 1 a \cdots \mathrm{H} 1 n 21,6=\mathrm{O} 71 a \cdots \mathrm{H} 1 n 25,7=\mathrm{O} 71 /$ $1 a \cdots \mathrm{H} 1 n 25,8=\mathrm{O} 71 / 3 a \cdots \mathrm{H} 1 n 23$, and $9=\mathrm{O} 71 a \cdots \mathrm{H} 1 n 23$. A similar plot for nitrate group $B$ is given as Fig. S9 in the supporting information. 
implies that they are weak hydrogen bonds (Jeffrey, 1997), which are hardly affected by the modulation. One would expect the molecules to close in, in order to strengthen the $\mathrm{N}-\mathrm{H} \cdots \mathrm{O}$ hydrogen bonds. However, this is prevented by actually the shortest interatomic distances between molecules.

Nitrate groups $A$ and $B$ are involved in $\mathrm{C}-\mathrm{H} \cdots \mathrm{O}$ interactions, which are shorter than the $\mathrm{N}-\mathrm{H} \cdots \mathrm{O}$ hydrogen bonds (Fig. 9). As short as $2.2 \AA$, these $\mathrm{H} \cdots \mathrm{O}$ distances are at the lower side of the reported $\mathrm{C}-\mathrm{H} \cdots \mathrm{O}$ hydrogen bonds (Desiraju, 1991; Desiraju et al., 1993; Steiner \& Desiraju, 1998; Desiraju \& Steiner, 2001; Munshi \& Row, 2005). It can thus be conjectured that even closer separations of these atoms would take them onto the repulsion part of the interaction curve, which would be energetically unfavourable. $\mathrm{C}-\mathrm{H} \cdots \mathrm{O}$ interactions rather than $\mathrm{N}-\mathrm{H} \cdots \mathrm{O}$ hydrogen bonds thus determine the packing in $\mathrm{Co}(\mathrm{sep})\left(\mathrm{NO}_{3}\right)_{3}$.

The shortest contacts between nitrate group $C$ and the sepulchrate cage are between the $\mathrm{O}$ atoms (atoms $\mathrm{O} 9$ ) and $\mathrm{H}$ atoms connected to the apical $\mathrm{C}$ atoms (atoms $\mathrm{H} 2 c 41$ through to $\mathrm{H} 2 c 46$ ) (Fig. 10). The range of $\mathrm{H} \cdots \mathrm{O}$ distances of $\sim 2.2-$ $2.5 \AA$ is similar to the distances of the $\mathrm{C}-\mathrm{H} \cdots \mathrm{O}$ contacts of nitrate groups $A$ and $B$ (Fig. 11). Finally, short intermolecular interatomic distances are found between $\mathrm{H}$ atoms attached to apical $\mathrm{C}$ atoms on neighbouring sepulchrate molecules (Fig. 12). Again, these contacts prevent a denser packing of the molecules.

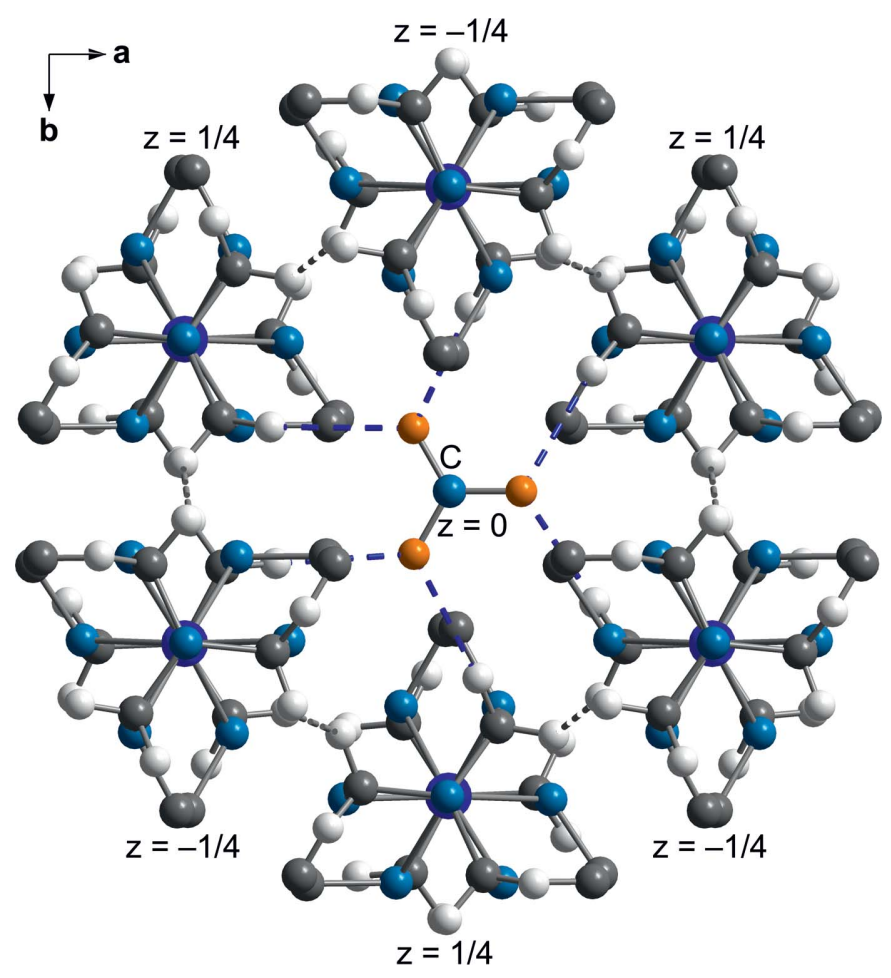

Figure 10

Projection onto ( 001 ) of nitrate group $C$ at $z=0$ and its six coordinating $\mathrm{Co}(\mathrm{sep})$ cages at $z= \pm 1 / 4$, as indicated. The shortest intermolecular contacts are $\mathrm{C}-\mathrm{H} \cdots \mathrm{O}$ hydrogen bonds (blue dashed lines) and $\mathrm{C}-$ $\mathrm{H} \cdots \mathrm{H}-\mathrm{C}$ contacts (grey dashed lines).

\section{Conclusions}

The 12-fold superstructure of $\Lambda$-cobalt(III) sepulchrate trinitrate at $T=95 \mathrm{~K}$ has successfully been described within the superspace approach. The superspace approach effectively removes correlations between crystallographically independent parameters of this high- $Z^{\prime}$ structure with $Z^{\prime}=12$.

A major difference between phase I - stable at ambient conditions - and phase IV - stable below $98 \mathrm{~K}$ - is the order of the nitrate group $C$ (Fig. 4). At room temperature nitrate group $C$ exhibits at least sixfold orientational disorder, while in phase IV this nitrate group is fully ordered in 12 different orientations in the 12-fold supercell (Fig. 7).

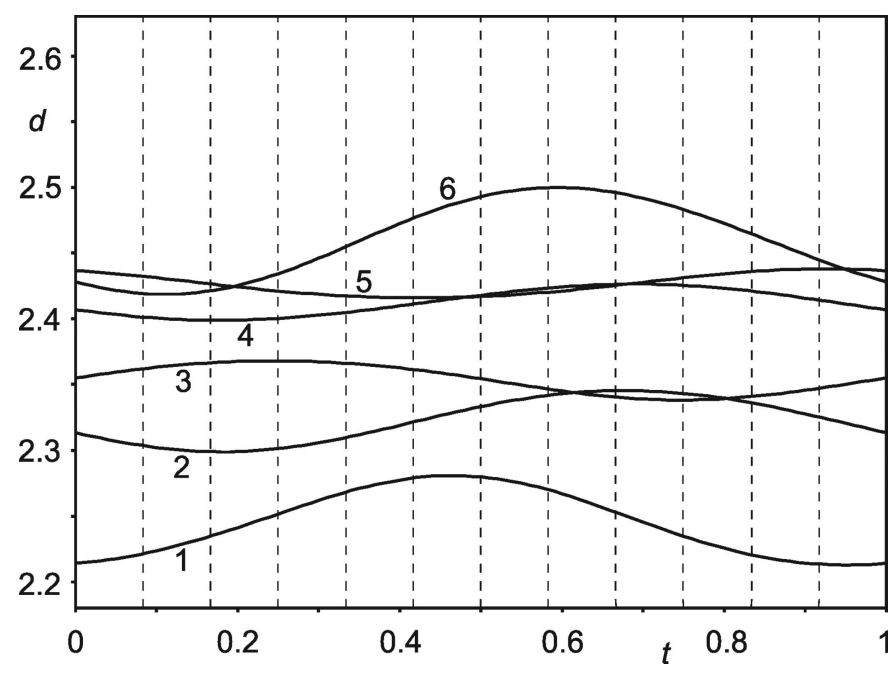

Figure 11

$t$-Plot of interatomic distances $d(\AA)$ between the $\mathrm{O}$ atom of nitrate group $C$ and $\mathrm{H}$ atoms of the sepulchate cage. Vertical, dashed lines indicate $t$ values corresponding to the distances in the 12-fold supercell. Six C$\mathrm{H} \cdots \mathrm{O}$ contacts are shown (cf. Fig. 1): $1=\mathrm{O} 91 a \cdots \mathrm{H} 2 c 42,2=\mathrm{O} 91 /$ $1 a \cdots \mathrm{H} 2 c 44,3=\mathrm{O} 91 / 3 a \cdots \mathrm{H} 2 c 43,4=\mathrm{O} 91 / 1 a \cdots \mathrm{H} 2 c 45,5=\mathrm{O} 91 /$ $3 a \cdot \mathrm{H} 2 c 46$, and $6=\mathrm{O} 91 a \cdots \mathrm{H} 2 c 41$.

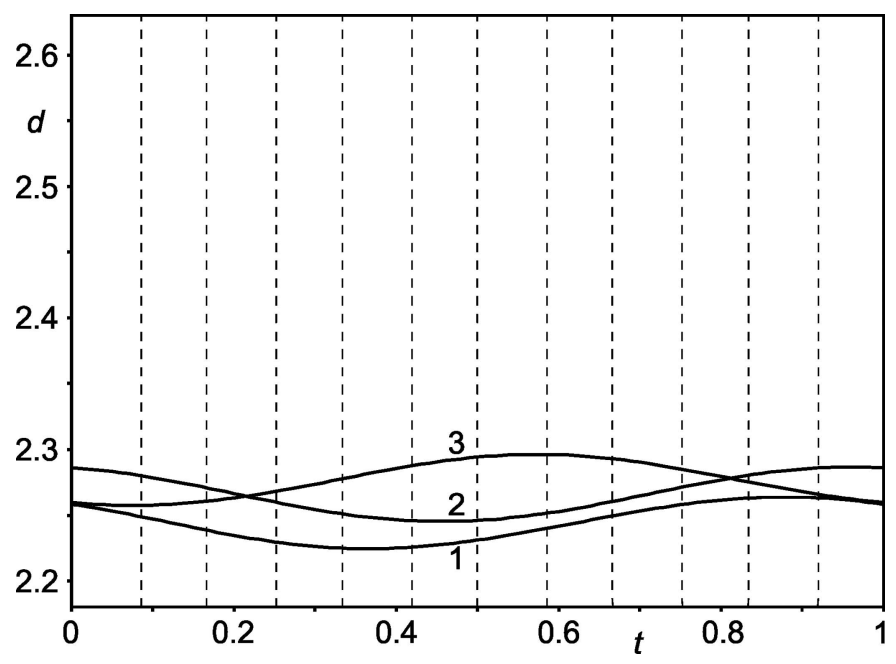

Figure 12

$t$-Plot of interatomic distances $d(\AA)$ between the $\mathrm{H}$ atoms attached to apical $\mathrm{C}$ atoms of neighbouring sepulchrate molecules. Vertical, dashed lines indicate $t$ values corresponding to the distances in the 12 -fold supercell. $1=\mathrm{H} 1 c 42 \cdots \mathrm{H} 1 c 43 ; 2=\mathrm{H} 1 c 41 \cdots \mathrm{H} 1 c 44 ; 3=\mathrm{H} 1 c 45 \cdots \mathrm{H} 1 c 46$. 
The origin of the modulation is argued to lie in the avoidance of repulsive interactions rather than hydrogen bonding between neighbouring molecules. The shortest interatomic contacts are $\mathrm{C}-\mathrm{H}$. . O weak hydrogen bonds (Figs. 9 and 11) and $\mathrm{C}-\mathrm{H} \cdots \mathrm{H}-\mathrm{C}$ intermolecular $\mathrm{H}$-atom contacts (Fig. 12). Intermolecular $\mathrm{N}-\mathrm{H} \cdots \mathrm{O}$ hydrogen bonds do exist (Fig. 9), but they are long and weak, apparently prevented from full development by the short intermolecular contacts described above.

\section{Acknowledgements}

We thank Professor Alan M. Sargeson for the donation of single crystals of $\mathrm{Co}(\operatorname{sep})\left(\mathrm{NO}_{3}\right)_{3}$. The assistance of Martin Tolkien in the diffraction experiments at beamline D3 of Hasylab at DESY, Hamburg, is gratefully acknowledged. Financial support has been obtained from the German Science Foundation (DFG) under grant number SCHO 830/3-1.

\section{References}

Arnold, H. (2006). International Tables for Crystallography, edited by T. Hahn, Vol. A, pp. 78-85. Chester: International Union of Crystallography.

Bacchi, A., Ferranti, F. \& Pelizzi, G. (1993). Acta Cryst. C49, 11631169.

Bacchi, A., Ferranti, F. \& Pelizzi, G. (1996). Acta Cryst. C52, 16241627.

Bernhardt, P. V., Chen, K.-I. \& Sharpe, P. C. (2006). J. Biol. Inorg. Chem. 11, 930-936.

Creaser, I. I., Geue, R. J., Harrowfield, J. M., Herlt, A. J., Sargeson, A. M., Snow, M. R. \& Springborg, J. (1982). J. Am. Chem. Soc. 104, 6016-6025.

Desiraju, G. R. (1991). Acc. Chem. Res. 24, 290-296.

Desiraju, G. R., Kashino, S., Coombs, M. M. \& Glusker, J. P. (1993). Acta Cryst. B49, 880-892.

Desiraju, G. R. \& Steiner, T. (2001). The Weak Hydrogen Bond in Structural Chemistry and Biology. Oxford University Press.

Dey, S., van Smaalen, S. \& Schönleber, A. (2016). Unpublished.

Dubiciki, L., Ferguson, J. \& Williamson, B. (1984). J. Phys. Chem. 88, 4254-4258.

Dubicki, L., Ferguson, J., Geue, R. J. \& Sargeson, A. M. (1980). Chem. Phys. Lett. 74, 393-397.

Gahan, L. R. \& Harrowfield, J. M. (2015). Polyhedron, 94, 1-51.

Garcia, R., Philp, E. F., Slawin, A. M. Z., Wright, P. A. \& Cox, P. A. (2001). Stud. Surf. Sci. Catal. 135, 157.
Görbitz, C. H., Törnroos, K. W. \& Day, G. M. (2012). Acta Cryst. B68, 549-557.

Hondow, N., Harowfield, J., Koutsantonis, G., Nealon, G. \& Saunders, M. (2012). Microporous Mesoporous Mater. 151, 264-270.

Janssen, T., Chapuis, G. \& de Boissieu, M. (2007). Aperiodic Crystals. From Modulated Phases to Quasicrystals. Oxford University Press.

Janssen, T., Janner, A., Looijenga-Vos, A. \& de Wolff, P. M. (2006). International Tables for Crystallography, edited by E. Prince, Vol. C, pp. 907-955. Chester: International Union of Crystallography.

Jeffrey, G. A. (1997). An Introduction to Hydrogen Bonding. Oxford University Press.

Larsen, F. K., Jørgensen, P., Hazell, R. G., Lebech, B., Thomas, R., Geue, R. J. \& Sargeson, A. M. (1988). Molecular Structure: Chemical Reactivity and Biological Activity, edited by J. J. Stezowski, J.-L. Huang \& M.-C. Shao, pp. 499-504. Oxford University Press.

Li, L., Wölfel, A., Schönleber, A., Mondal, S., Schreurs, A. M. M., Kroon-Batenburg, L. M. J. \& van Smaalen, S. (2011). Acta Cryst. B67, 205-217.

Munshi, P. \& Guru Row, T. N. (2005). J. Phys. Chem. A, 109, 659672.

Palatinus, L. \& Chapuis, G. (2007). J. Appl. Cryst. 40, 786-790.

Petricek, V., Dusek, M. \& Palatinus, L. (2014). Z. Kristallogr. 229, 345-352.

Pina, F., Mulazzani, Q. G., Venturi, M., Ciano, M. \& Balzani, V. (1985). Inorg. Chem. 24, 848-851.

Pinheiro, C. B. \& Abakumov, A. M. (2015). IUCrJ, 2, 137-154.

Ritzert, N. L., Rodríguez-López, J., Tan, C. \& Abruña, H. D. (2013). Langmuir, 29, 1683-1694.

Schomaker, V. \& Trueblood, K. N. (1968). Acta Cryst. B24, 63-76.

Schönleber, A. (2011). Z. Kristallogr. 226, 499-517.

Schönleber, A., van Smaalen, S. \& Larsen, F. K. (2010). Acta Cryst. C66, m107-m109.

Schreurs, A. M. M., Xian, X. \& Kroon-Batenburg, L. M. J. (2010). J. Appl. Cryst. 43, 70-82.

Sheldrick, G. M. (2008). SADABS. University of Göttingen, Germany.

Steed, J. W. (2016). The High $Z^{\prime}$ Structure Data Base, http:// zprime.co.uk/database.

Steed, K. M. \& Steed, J. W. (2015). Chem. Rev. 115, 2895-2933.

Steiner, T. \& Desiraju, G. R. (1998). Chem. Commun. pp. 891-892.

Stokes, H. T., Campbell, B. J. \& van Smaalen, S. (2011). Acta Cryst. A67, 45-55.

van Smaalen, S. (2012). Incommensurate Crystallography. Oxford University Press.

van Smaalen, S., Campbell, B. J. \& Stokes, H. T. (2013). Acta Cryst. A69, 75-90. 